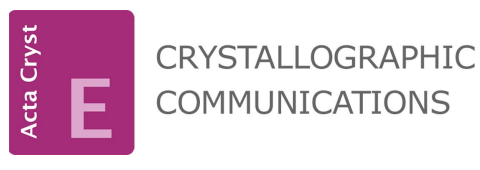

ISSN 2056-9890

Received 1 July 2015

Accepted 12 July 2015

Edited by W. T. A. Harrison, University of Aberdeen, Scotland

Keywords: crystal structure; polyhalogenated benzene; halogen bond; bromine; iodine

CCDC references: $1412444 ; 1412445$ Supporting information: this article has supporting information at journals.iucr.org/e

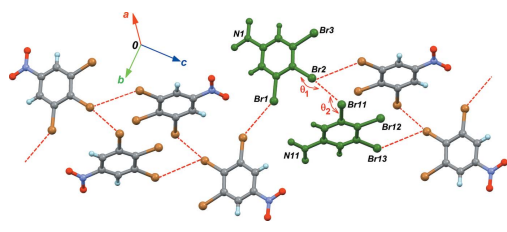

OPEN $\odot$ ACCESS

\section{Anomalous halogen bonds in the crystal structures of 1,2,3-tribromo-5-nitrobenzene and 1,3-dibromo- 2-iodo-5-nitrobenzene}

\author{
José A. Romero, ${ }^{a}$ Gerardo Aguirre Hernández ${ }^{\mathrm{a}}$ and Sylvain Bernès ${ }^{\mathrm{b} *}$
}

${ }^{a}$ Centro de Graduados e Investigación en Química, Instituto Tecnológico de Tijuana, Apdo. Postal 1166, 22510 Tijuana, B.C., Mexico, and ${ }^{\mathbf{b}}$ Instituto de Física, Benemérita Universidad Autónoma de Puebla, Av. San Claudio y 18 Sur, 72570 Puebla, Pue., Mexico. *Correspondence e-mail: sylvain_bernes@hotmail.com

The title trihalogenated nitrobenzene derivatives, $\mathrm{C}_{6} \mathrm{H}_{2} \mathrm{Br}_{3} \mathrm{NO}_{2}$ and $\mathrm{C}_{6} \mathrm{H}_{2} \mathrm{Br}_{2} \mathrm{INO}_{2}$, crystallize in triclinic and monoclinic cells, respectively, with two molecules per asymmetric unit in each case. The asymmetric unit of the tribromo compound features a polarized $\mathrm{Br}^{\delta+} \ldots \mathrm{Br}^{\delta-}$ intermolecular halogen bond. After substitution of the $\mathrm{Br}$ atom in the para position with respect to the nitro group, the network of $X \cdots X$ halogen contacts is reorganized. Two intermolecular polarized halogen bonds are then observed, which present the uncommon polarization $\mathrm{Br}^{\delta+} \ldots \mathrm{I}^{\delta-}$ : the more electronegative site $(\mathrm{Br})$ behaves as a donor and the less electronegative site (I) as an acceptor for the charge transfer.

\section{Chemical context}

Within the large class of non-covalent interactions studied in chemical crystallography, halogen bonds are of special interest in crystal engineering. The stabilizing interaction between a halogen atom and a Lewis base, $X \cdots \mathrm{B}$, shares many aspects with classical hydrogen bonds, but is more directional. On the other hand, halogen contacts $X \cdots X$ are more difficult to conceptualize (Wang et al., 2014), for instance because the charge transfer in the $\mathrm{Br} \cdot \mathrm{Br}$ contact is not as obvious as in hydrogen bonds. Evidence supporting the importance of this topic is the recent organization of an international meeting dedicated to halogen bonding (Erdelyi, 2014).<smiles>O=[N+]([O-])c1cc(Br)c(Br)c(Br)c1</smiles>

(I)<smiles>[3H]c1c(Br)cc([N+](=O)[O-])cc1Br</smiles>

(II)
In this context, we are engaged in the synthesis and structural characterization of a series of halogen-substituted nitrobenzenes. The present communication describes two closely related compounds in the series, which differ only by the halogen atom substituting at the ring position para to the nitro group. Despite the small chemical modification, the resulting crystal structures are very different, as a consequence of a different network of halogen bonds. 


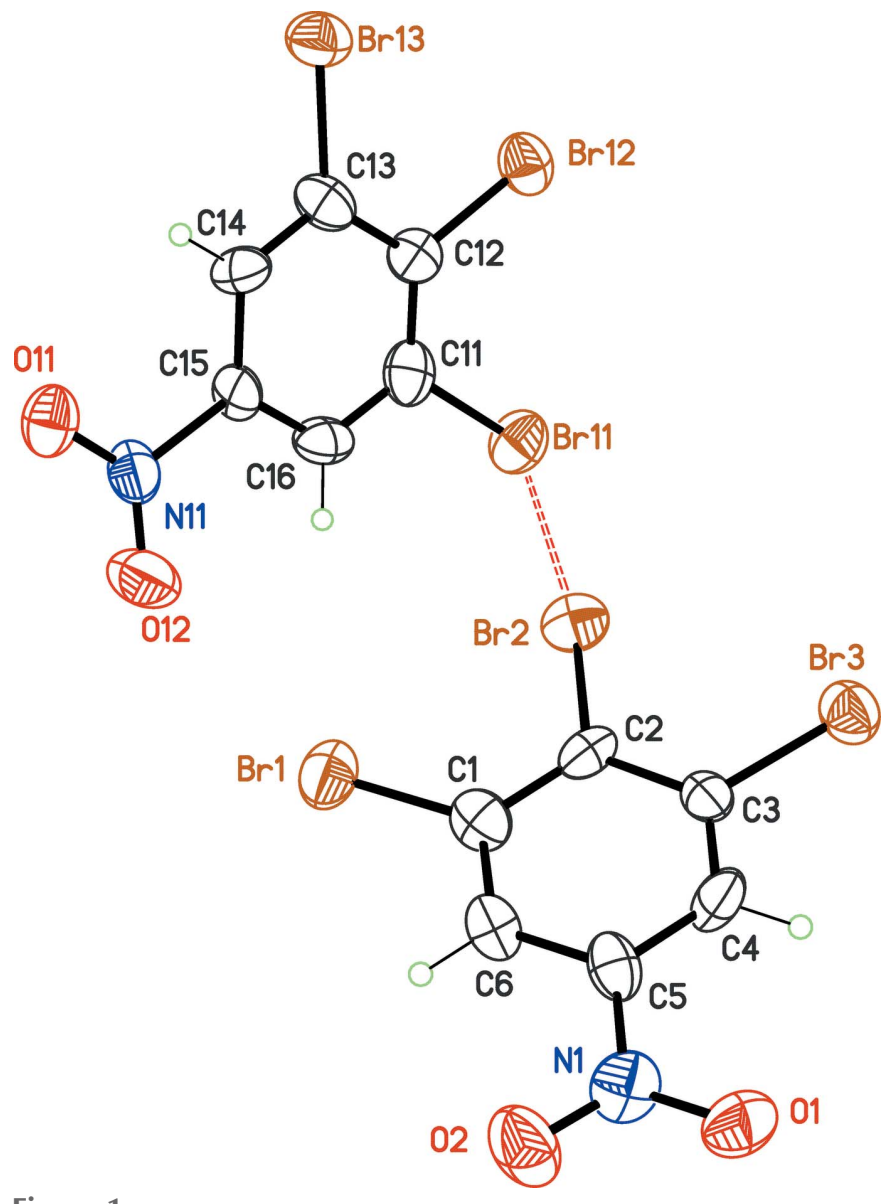

Figure 1

The asymmetric unit of (I), with displacement ellipsoids at the $30 \%$ probability level. The dashed bond connecting the independent molecules is a type-II halogen bond.

\section{Structural commentary}

Both compounds crystallize with two molecules in the asymmetric unit, but in different space groups. The tribromo derivative, (I, Fig. 1), is a $P \overline{1}$ crystal isomorphous to the chloro analogue (Bhar et al., 1995), although the unit-cell parameters are significantly larger for (I) compared to the chloro compound: the cell volume is increased by more than $7 \%$. In the present work, we retained the Niggli reduced triclinic cell $(a<b<c)$, while Bhar et al. used a non-reduced cell. Moreover, the asymmetric unit content was defined in order to emphasize the strongest $\mathrm{Br} \cdots \mathrm{Br}$ bond in (I). The bromo-iodo derivative (II, Fig. 2) crystallizes in the monoclinic system and, in that case, the standard setting was used for space group $P 2_{1} / c$.

The $\mathrm{C}-$ halogen bond lengths are as expected. In (I), $\mathrm{C}-\mathrm{Br}$ distances are in the range $1.821(12)-1.886$ (11) $\AA$, slightly shorter than $\mathrm{C}-\mathrm{Br}$ bond lengths observed in hexabromobenzene, $1.881 \AA$ ( $T=100 \mathrm{~K}$; Reddy et al., 2006) or $1.871 \AA$ (synchrotron study, $T=100 \mathrm{~K}$; Brezgunova et al., 2012). In (II), $\mathrm{C}-\mathrm{Br}$ bond lengths are longer, 1.875 (13) to 1.895 (14) $\AA$, while the C-I bond lengths, 2.088 (12) and 2.074 (14) $\AA$, may be compared to bonds in hexaiodobenzene, $2.109 \AA(T=$ $100 \mathrm{~K}$; Ghosh et al., 2007) or 1,2,3-triiodobenzene, $2.090 \AA$ ( $T$

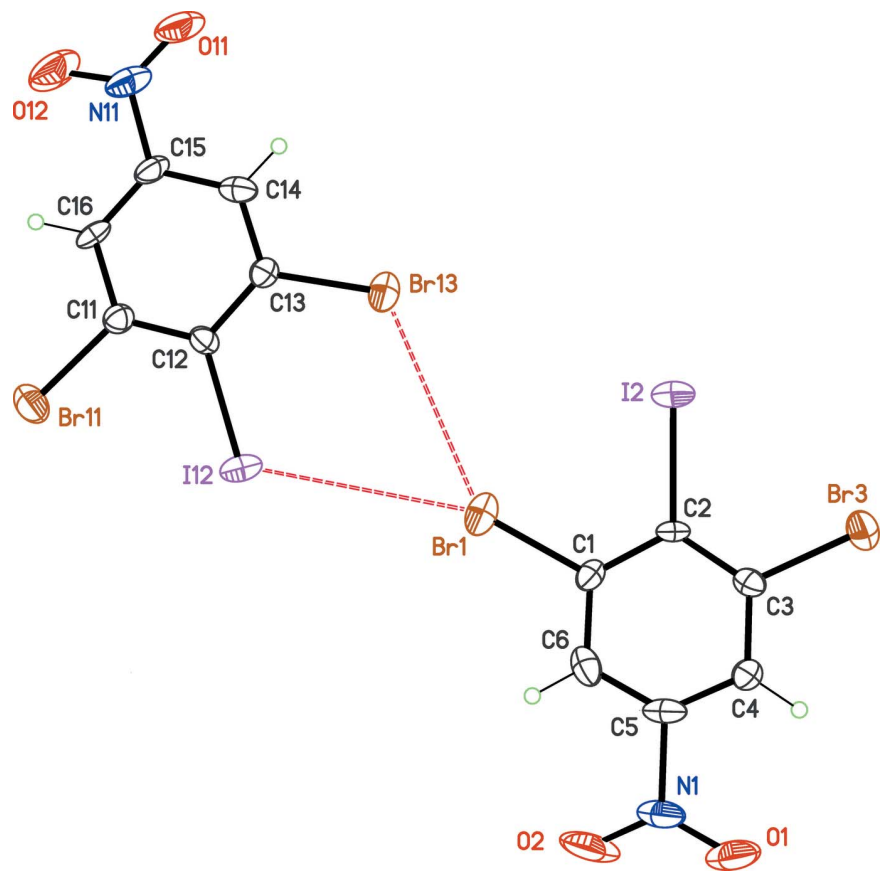

Figure 2

The asymmetric unit of (II), with displacement ellipsoids at the $30 \%$ probability level. The dashed bonds connecting the independent molecules are halogen contacts.

$=223 \mathrm{~K}$, Novak \& Li, 2007). Indeed, differences in bond lengths between perhalogenated and trihalogenated derivatives are within experimental errors, and the substitution of the 5-position by the nitro electron-withdrawing group in (I) and (II) has probably little influence on these bonds.

The important feature in these halogenated molecules is rather the possibility of steric repulsion between vicinal halogen atoms, which is related to the reduction of endocyclic angles. Regarding this point, it is worth reading the Acta $E$ article about 1,2,3-triiodobenzene (Novak \& Li, 2007). As in polyiodo derivatives, intramolecular steric crowding between the halogen atoms in (I) and (II) is offset by benzene ring distortion. As a consequence, the $\mathrm{C} 1-\mathrm{C} 2-\mathrm{C} 3$ and equivalent $\mathrm{C} 11-\mathrm{C} 12-\mathrm{C} 13$ angles are systematically less than $120^{\circ}$ : 116.2 (11) and $118.8(13)^{\circ}$ in (I); 118.1 (12) and $117.3(13)^{\circ}$ in (II). Again, the nitro group has little influence on intramolecular halogen $\cdots$ halogen contacts. For instance, in 1,3dibromo-2-iodobenzene, the $\mathrm{C} 1-\mathrm{C} 2-\mathrm{C} 3$ angle is $118.0^{\circ}$ (Schmidbaur et al., 2004), very close to that observed in (II), which presents the same halogen substitution.

The 5-nitro substituent is almost conjugated with the benzene nucleus in (I): the dihedral angle between the $\mathrm{NO}_{2}$ plane and the benzene ring is $6(2)$ and $1(2)^{\circ}$ for each independent molecule. For (II), twisting of the $\mathrm{NO}_{2}$ groups is more significant, with dihedral angles of $10(1)$ and $7(1)^{\circ}$. This near planar conformation is identical to that observed for 1,2,3trichloro-5-nitrobenzene (Bhar et al., 1995), but contrasts with the twisted conformation observed in perhalogenated nitrobenzene derivatives: pentachloronitrobenzene (twist angle of $\mathrm{NO}_{2}: 62^{\circ}$; Tanaka et al., 1974) and 1-bromo-2,3,5,6-tetrafluoro4-nitrobenzene (twist angle of $\mathrm{NO}_{2}: 41.7(3)^{\circ}$; Stein et al., 
Table 1

Halogen-bond geometry $\left(\AA{ }^{\circ}{ }^{\circ}\right)$ for $(\mathrm{I})$.

\begin{tabular}{lllll}
\hline$X_{1} \cdots X_{2}$ & $d$ & $\theta_{1}$ & $\theta_{2}$ & bond type \\
\hline $\mathrm{Br} 2 \cdots \mathrm{Br} 11$ & $3.642(3)$ & $165.2(5)$ & $82.3(5)$ & II-polarized \\
$\mathrm{Br} 1 \cdots \mathrm{Br} 1^{\mathrm{i}}$ & $3.731(4)$ & $133.3(4)$ & $133.3(4)$ & I-unpolarized \\
$\mathrm{Br} 2 \cdots \mathrm{Br} 13^{\mathrm{ii}}$ & $3.781(3)$ & $126.8(4)$ & $129.6(4)$ & I-unpolarized \\
\hline
\end{tabular}

Notes: $d=$ separation $X_{1} \cdots X_{2} ; \theta_{1}=$ angle $\mathrm{C}-X_{1} \cdots X_{2} ; \theta_{2}=$ angle $X_{1} \cdots X_{2}-\mathrm{C}$. For halogen bond types, see: Reddy et al. (2006). Symmetry codes: (i) $-x, 1-y,-z$; (ii) $-x$, $-y, 1-z$.

Table 2

Halogen-bond geometry $\left(\AA{ }^{\circ}\right)$ for (II).

\begin{tabular}{lllll}
\hline$X_{1} \cdots X_{2}$ & $d$ & $\theta_{1}$ & $\theta_{2}$ & bond type \\
\hline $\mathrm{Br} 1 \cdots \mathrm{I} 12$ & $3.813(2)$ & $161.2(4)$ & $117.2(4)$ & II-polarized \\
$\mathrm{I} 2 \cdots \mathrm{Br} 11^{\mathrm{i}}$ & $3.893(2)$ & $116.6(4)$ & $161.8(4)$ & II-polarized \\
$\mathrm{Br} 1 \cdots \mathrm{Br} 13$ & $3.787(2)$ & $142.8(4)$ & $122.9(4)$ & I-unpolarized \\
$\mathrm{Br}^{1} \cdots \mathrm{Br} 3^{\mathrm{ii}}$ & $3.858(2)$ & $143.9(4)$ & $124.4(4)$ & I-unpolarized \\
\hline
\end{tabular}

Notes: $d=$ separation $X_{1} \cdots X_{2} ; \theta_{1}=$ angle $\mathrm{C}-X_{1} \cdots X_{2} ; \theta_{2}=$ angle $X_{1} \cdots X_{2}-\mathrm{C}$. For halogen bond types, see: Reddy et al. (2006). Symmetry codes: (i) $1+x, y, z$; (ii) $-1+x, y$, $z$.

2011). It thus seems clear that twisting of the nitro group with respect to the benzene ring in nitrobenzene derivatives is a direct consequence of intramolecular crowding with ortho substituents. For 1,2,3-halogenated-5-nitrobenzenes such as (I) and (II), a planar conformation should be expected as a rule.

\section{Supramolecular features}

The crystal structures are directed by intermolecular weak halogen bonds, also known as type-II interactions in the Desiraju classification scheme (Reddy et al., 2006). Such a bond is present in the asymmetric unit of (I), between $\mathrm{Br} 2$ and Br11 (Fig. 3). The type-II arrangement is characterized by angles $\theta_{1}=\mathrm{C} 2-\mathrm{Br} 2 \cdots \mathrm{Br} 11$ and $\theta_{2}=\mathrm{C} 11-\mathrm{Br} 11 \cdots \mathrm{Br} 2$, which

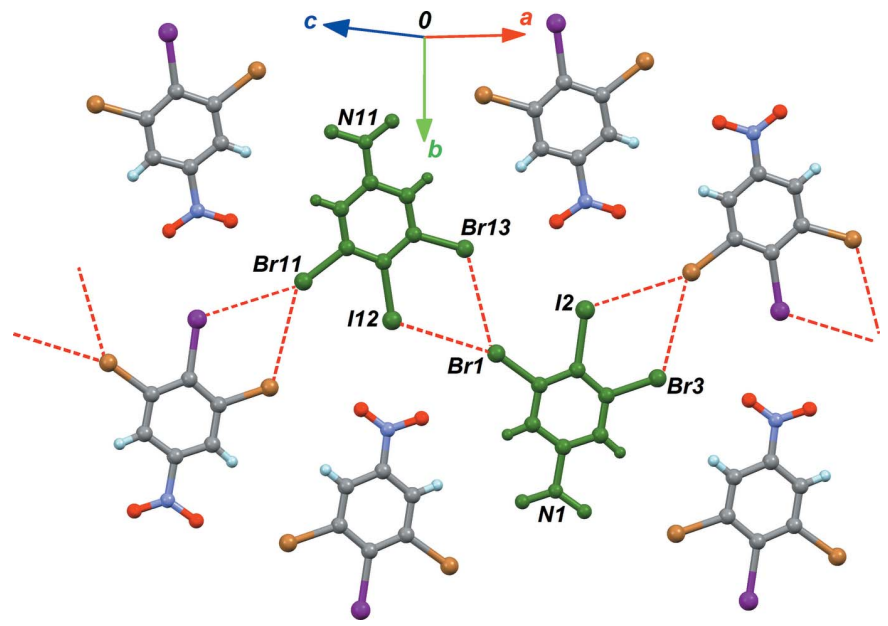

Figure 4

Part of the crystal structure of (II), emphasizing the halogen bonds (dashed lines). The green molecules correspond to the asymmetric unit.

should be close to 180 and $90^{\circ}$, respectively. For (I), observed angles are $\theta_{1}=165.2(5)^{\circ}$ and $\theta_{2}=82.3(5)^{\circ}$. The crystal packing thus polarizes the involved halogen atoms, forming the halogen bond $\mathrm{Br} 2^{\delta+} \ldots \mathrm{Br} 11^{\delta-}$. This dimolecular polar unit is connected via inversion centers to neighboring units in the cell, forming $\mathrm{C}-\mathrm{H} \cdots \mathrm{Br}$ hydrogen bonds, and $\mathrm{O} \cdots \mathrm{Br}$ contacts. This packing motif induces secondary halogen..halogen contacts, which are clearly unpolarized. These type-I interactions are characterized by angles $\theta_{1} \simeq \theta_{2}$ (Table 1 , entries 2 and 3) and display larger $\mathrm{Br} \cdot \mathrm{Br}$ separations compared to the polarized halogen bond (entry 1), in which electrostatic forces bring the atoms into close contact.

The substitution of one $\mathrm{Br}$ atom by I, to form crystal (II), changes dramatically the packing structure, affording a more complex network of halogen contacts (Fig. 4 and Table 2). Within the asymmetric unit, the type-II polarized contact is Br1‥I12 (Table 2, entry 1). However, $\theta$ angles for this bond deviate from ideal values, and, surprisingly, the bond is

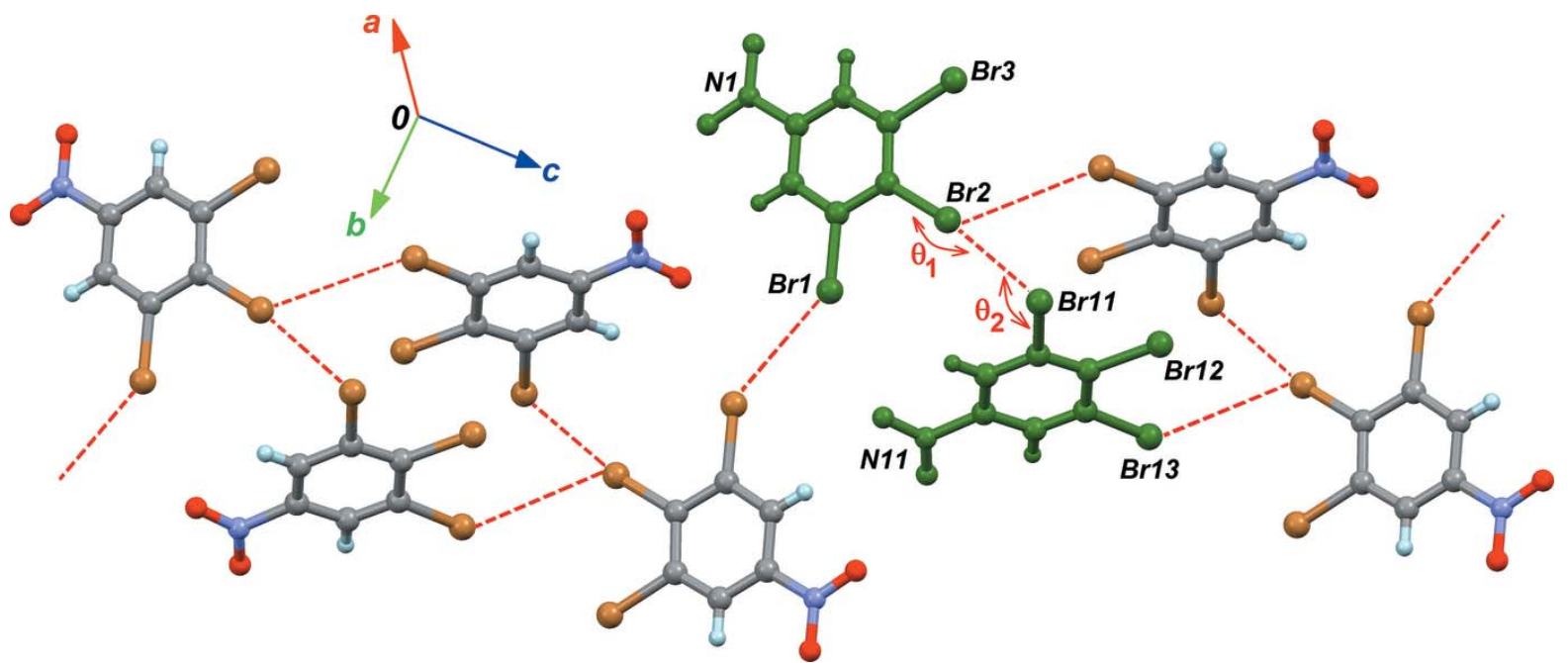

Figure 3

Part of the crystal structure of (I), emphasizing the halogen bonds (dashed lines). The green molecules correspond to the asymmetric unit. 
polarized in the wrong way, $\mathrm{Br}^{\delta+} \ldots \mathrm{I}^{\delta-}$. The opposite polarization was expected for this bond, due to the lower electronegativity and higher polarizability of iodine compared to bromine. The other significant contact observed in the asymmetric unit is a $\mathrm{Br} \cdots \mathrm{Br}$ unpolarized contact. The network of halogen bonds is expanded in the [100] direction by Br11, which gives a bifurcated contact with $\mathrm{I} 2$ and $\mathrm{Br} 3$ (Table 2, entries 2 and 4). One contact is polarized, with the polarization, once again, oriented in the unexpected way, $\mathrm{I} 2^{\delta-} \ldots \mathrm{Br} 11^{\delta+}$. These anomalous halogen bonds are not present in other mixed halogen derivatives. Indeed, in 1,3-dibromo-2iodobenzene (Schmidbaur et al., 2004), the iodine atom is not engaged in halogen bonding.

\section{Database survey}

The current release of the CSD (Version 5.36 with all updates; Groom \& Allen, 2014), contains many structures of halogensubstituted nitrobenzene, with $\mathrm{Cl}$ (e.g. Bhar et al., 1995; Tanaka et al., 1974), Br (e.g. Olaru et al., 2014), and I (Thalladi et al., 1996). This series is completed with nitrophenol derivatives, for example 2,3-difluoro-4-iodo-6-nitrophenol (Francke et al., 2010). Structures of pentachlorophenol (Brezgunova $e t$ al., 2012) and pentabromophenol (Betz et al., 2008; Brezgunova et al., 2012) are also available.

Regarding poly- and per-halogenated benzene structures, an impressive series of 23 compounds has been described, including $\mathrm{Cl}, \mathrm{Br}$, I and $\mathrm{Me}$ as substituents, generating a variety

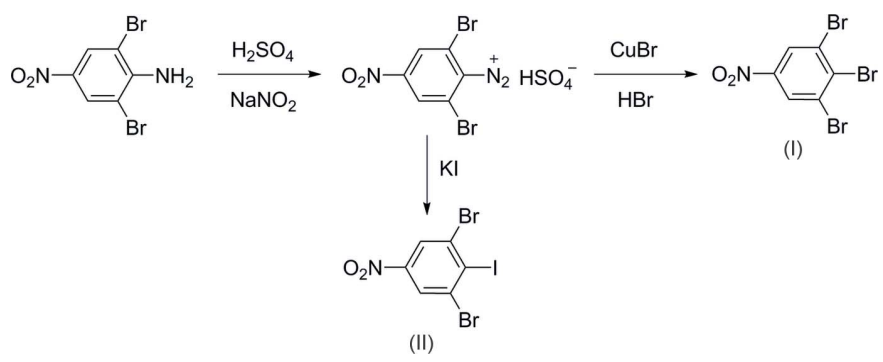

Figure 5

Synthetic scheme for (I) and (II).

of molecular symmetries (Reddy et al., 2006). The structure of $D_{6 \mathrm{~h}}$-perhalogenated benzene has been reported with $\mathrm{F}$ (Shorafa et al., 2009), Cl (Brown \& Strydom, 1974; Reddy et al., 2006), Br (Baharie \& Pawley, 1979; Reddy et al., 2006; Brezgunova et al., 2012) and I (Ghosh et al., 2007). The former is a $Z^{\prime}=2$ crystal, while others are $Z^{\prime}=1$ crystals.

\section{Synthesis and crystallization}

Compounds (I) and (II) were synthesized from 2,6-dibromo-4nitroaniline (Bryant et al., 1998), as depicted in Fig. 5.

Synthesis of (I). A solution of 2,6-dibromo-4-nitroaniline $(1.0 \mathrm{~g}, 3.38 \mathrm{mmol})$ in acetic acid $(3 \mathrm{ml})$ was cooled to $278 \mathrm{~K}$, and concentrated $\mathrm{H}_{2} \mathrm{SO}_{4}(7 \mathrm{ml})$ was carefully added under stirring. While ensuring that the temperature was still below $278 \mathrm{~K}, \mathrm{NaNO}_{2}(0.708 \mathrm{~g}, 10.26 \mathrm{mmol})$ was added in one batch. The reaction was stirred at this temperature for $2 \mathrm{~h}$ to afford

Table 3

Experimental details.

(I)

Crystal data

Chemical formula

$M_{\mathrm{r}}$

Crystal system, space group

Temperature $(\mathrm{K})$

$a, b, c(\AA)$

$\alpha, \beta, \gamma\left({ }^{\circ}\right)$

$V\left(\AA^{3}\right)$

$Z$

Radiation type

$\mu\left(\mathrm{mm}^{-1}\right)$

Crystal size (mm)

Data collection

Diffractometer

Absorption correction

$T_{\min }, T_{\max }$

No. of measured, independent and observed

$[I>2 \sigma(I)]$ reflections

$R_{\text {int }}$

$(\sin \theta / \lambda)_{\max }\left(\AA^{-1}\right)$

Refinement

$R\left[F^{2}>2 \sigma\left(F^{2}\right)\right], w R\left(F^{2}\right), S$

No. of reflections

No. of parameters

$\mathrm{H}$-atom treatment

$\Delta \rho_{\max }, \Delta \rho_{\min }\left(\mathrm{e} \AA^{-3}\right)$

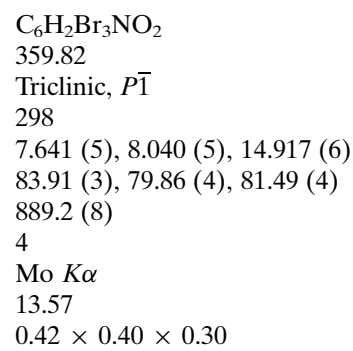

(II)

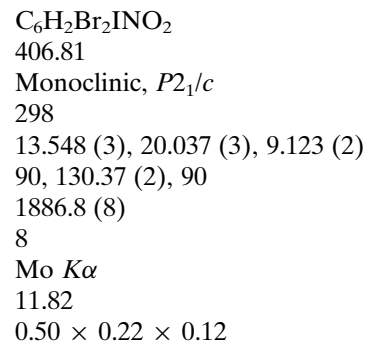

Bruker P4

Part of the refinement model $(\Delta F)$ (Walker \& Stuart, 1983)

$0.0002,0.001$

$6070,3141,1503$

0.120

0.596

Bruker P4
$\psi$ scan (XSCANS; Bruker, 1997)
$0.429,0.988$
$5716,5407,1968$

0.058
0.703

$0.061,0.153,0.95$
5407
218
H-atom parameters constrained
$0.84,-0.84$

Computer programs: XSCANS (Bruker, 1997), SHELXS2014 (Sheldrick, 2008), SHELXL2014 (Sheldrick, 2015) and Mercury (Macrae et al., 2008). 
the diazonium salt. An aqueous solution $(17.67 \mathrm{ml})$ of $\mathrm{CuBr}$ $(4.95 \mathrm{~g}, 34.54 \mathrm{mmol})$ and $47 \% \mathrm{HBr}(17.67 \mathrm{ml})$ was warmed to $343 \mathrm{~K}$, and the diazotization solution previously prepared was added in one batch with stirring. The mixture was kept at $343 \mathrm{~K}$ for $1 \mathrm{~h}$, and then left to cool overnight. The reaction was neutralized with $\mathrm{NaOH}$ and extracted with $\mathrm{CH}_{2} \mathrm{Cl}_{2}(3 \times$ $30 \mathrm{ml}$ ). The resulting solution was concentrated under vacuum and the crude material was purified by flash chromatography (petroleum ether $/ \mathrm{CH}_{2} \mathrm{Cl}_{2} 8 / 2, R_{\mathrm{f}}=0.49$ ) to give (I). Crystals were obtained by slow evaporation of a methanol/ethyl ether solution (yield: $0.952 \mathrm{~g}, 2.65 \mathrm{mmol}, 78 \%$ ). m.p. 380-382 K. IR $\left(\mathrm{KBr}, \mathrm{cm}^{-1}\right): 3090(\mathrm{Ar}-\mathrm{H}) ; 1583(\mathrm{C}=\mathrm{C}) ; 1526,1342(\mathrm{~N}=\mathrm{O})$; $738(\mathrm{C}-\mathrm{Br}) .{ }^{1} \mathrm{H}-\mathrm{NMR}\left(600 \mathrm{MHz}, \mathrm{CDCl}_{3}\right): \delta 8.43(s, \mathrm{H}-4, \mathrm{H}-6)$. ${ }^{13} \mathrm{C}-\mathrm{NMR}\left(150 \mathrm{MHz}, \mathrm{CDCl}_{3}\right): \delta 146.8,135.7,127.0,126.9$, 126.8. EIMS $\mathrm{m} / z$ : $\left[M^{+}\right] 357(34),\left[M^{+}+2\right] 359(7),\left[M^{+}+4\right]$ $361(100),\left[M^{+}+6\right] 363(36)\left[M^{+}-\mathrm{NO}_{2}\right] 311(12)$.

Synthesis of (II). A solution of 2,6-dibromo-4-nitroaniline $(1.0 \mathrm{~g}, 3.38 \mathrm{mmol})$ in acetic acid $(3 \mathrm{ml})$ was cooled to $278 \mathrm{~K}$ in an ice-salt bath, and concentrated $\mathrm{H}_{2} \mathrm{SO}_{4}(3 \mathrm{ml})$ was carefully added under stirring. While ensuring that the temperature was still below $278 \mathrm{~K}, \mathrm{NaNO}_{2}(0.242 \mathrm{~g}, 3.516 \mathrm{mmol})$ was added in one batch. The reaction was stirred at this temperature for $30 \mathrm{~min}$ to afford the diazonium salt. An aqueous solution $(10 \mathrm{ml})$ of KI $(5.635 \mathrm{~g}, 33.95 \mathrm{mmol})$ was prepared, and the diazotization solution previously prepared was added in one batch. The mixture was then further stirred for $1 \mathrm{~h}$. The reaction was neutralized with $\mathrm{NaOH}$, extracted with $\mathrm{CH}_{2} \mathrm{Cl}_{2}$ $(3 \times 30 \mathrm{ml})$, and concentrated under vacuum. The crude material was purified by flash chromatography (petroleum ether $/ \mathrm{CH}_{2} \mathrm{Cl}_{2} 4 / 1, R_{\mathrm{f}}=0.31$ ) to give (II). Crystals were obtained by slow evaporation of an acetone/methanol/ $/ \mathrm{CH}_{2} \mathrm{Cl}_{2}$ solution (yield: $1.21 \mathrm{~g}, 2.98 \mathrm{mmol}, 88 \%$ ). m.p. 415-417 K. IR $\left(\mathrm{KBr}, \mathrm{cm}^{-1}\right)$ : $3010(\mathrm{Ar}-\mathrm{H}) ; 1620,1516(\mathrm{C}=\mathrm{C}) ; 1336(\mathrm{~N}=\mathrm{O})$. ${ }^{1} \mathrm{H}-\mathrm{NMR}\left(600 \mathrm{MHz}, \mathrm{CDCl}_{3}\right): \delta 8.38(s, \mathrm{H}-4, \mathrm{H}-6) .{ }^{13} \mathrm{C}-\mathrm{NMR}$ $\left(150 \mathrm{MHz}, \mathrm{CDCl}_{3}\right): \delta 146.1,142.4,127.4,124.1$. EIMS $m / z$ : $\left[M^{+}\right] 405$ (42), [ $\left.M^{+}+2\right] 407$ (100), [ $\left.M^{+}+4\right] 409$ (48).

\section{Refinement}

Crystal data, data collection and structure refinement details for (I) and (II) are summarized in Table 3. The absorption correction for (I) was challenging, and eventually carried out by applying DIFABS on the complete isotropic model (Walker \& Stuart, 1983). In the case of (II), measured $\psi$-scans were used. $\mathrm{H}$ atoms were refined as riding to their carrier $\mathrm{C}$ atoms, with $\mathrm{C}-\mathrm{H}$ bond lengths fixed at $0.93 \AA$ and with $U_{\text {iso }}(\mathrm{H})=1.2 U_{\text {eq }}($ carrier atom $)$.

\section{Acknowledgements}

We acknowledge the contribution of Angélica Navarrete to the synthesis of the reported compounds.

\section{References}

Baharie, E. \& Pawley, G. S. (1979). Acta Cryst. A35, 233-235.

Betz, R., Klüfers, P. \& Mayer, P. (2008). Acta Cryst. E64, o1921.

Bhar, A., Aune, J. P., Benali-Cherif, N., Benmenni, L. \& Giorgi, M. (1995). Acta Cryst. C51, 256-260.

Brezgunova, M. E., Aubert, E., Dahaoui, S., Fertey, P., Lebègue, S., Jelsch, C., Ángyán, J. G. \& Espinosa, E. (2012). Cryst. Growth Des. 12, 5373-5386.

Brown, G. M. \& Strydom, O. A. W. (1974). Acta Cryst. B30, 801-804.

Bruker (1997). XSCANS. Bruker Analytical X-ray Instruments Inc., Madison, Wisconsin, USA.

Bryant, R., James, S. C., Norman, N. C. \& Orpen, A. G. (1998). Acta Cryst. C54, 1113-1115.

Erdelyi, M. (2014). Nat. Chem. 6, 762-764.

Francke, R., Schnakenburg, G. \& Waldvogel, S. R. (2010). Eur. J. Org. Chem. pp. 2357-2362.

Ghosh, S., Reddy, C. M. \& Desiraju, G. R. (2007). Acta Cryst. E63, o910-0911.

Groom, C. R. \& Allen, F. H. (2014). Angew. Chem. Int. Ed. 53, 662671.

Macrae, C. F., Bruno, I. J., Chisholm, J. A., Edgington, P. R., McCabe, P., Pidcock, E., Rodriguez-Monge, L., Taylor, R., van de Streek, J. \& Wood, P. A. (2008). J. Appl. Cryst. 41, 466-470.

Novak, I. \& Li, D. (2007). Acta Cryst. E63, o438-o439.

Olaru, M., Beckmann, J. \& Raţ, C. I. (2014). Organometallics, 33, 3012-3020.

Reddy, C. M., Kirchner, M. T., Gundakaram, R. C., Padmanabhan, K. A. \& Desiraju, G. R. (2006). Chem. Eur. J. 12, 2222-2234.

Schmidbaur, H., Minge, O. \& Nogai, S. (2004). Z. Naturforsch. Teil B, 59, 264-268.

Sheldrick, G. M. (2008). Acta Cryst. A64, 112-122.

Sheldrick, G. M. (2015). Acta Cryst. C71, 3-8.

Shorafa, H., Mollenhauer, D., Paulus, B. \& Seppelt, K. (2009). Angew. Chem. Int. Ed. 48, 5845-5847.

Stein, M., Schwarzer, A., Hulliger, J. \& Weber, E. (2011). Acta Cryst. E67, o1655.

Tanaka, I., Iwasaki, F. \& Aihara, A. (1974). Acta Cryst. B30, 15461549.

Thalladi, V. R., Goud, B. S., Hoy, V. J., Allen, F. H., Howard, J. A. K. \& Desiraju, G. R. (1996). Chem. Commun. pp. 401-402.

Walker, N. \& Stuart, D. (1983). Acta Cryst. A39, 158-166.

Wang, C., Danovich, D., Mo, Y. \& Shaik, S. (2014). J. Chem. Theory Comput. 10, 3726-3737. 


\section{supporting information}

Acta Cryst. (2015). E71, 960-964 [https://doi.org/10.1107/S2056989015013377]

Anomalous halogen bonds in the crystal structures of 1,2,3-tribromo-5-nitrobenzene and 1,3-dibromo-2-iodo-5-nitrobenzene

José A. Romero, Gerardo Aguirre Hernández and Sylvain Bernès

Computing details

For both compounds, data collection: XSCANS (Bruker, 1997); cell refinement: XSCANS (Bruker, 1997); data reduction: XSCANS (Bruker, 1997); program(s) used to solve structure: SHELXS2014 (Sheldrick, 2008); program(s) used to refine structure: SHELXL2014 (Sheldrick, 2015); molecular graphics: Mercury (Macrae et al., 2008); software used to prepare material for publication: SHELXL2014 (Sheldrick, 2015).

(I) 1,2,3-Tribromo-5-nitrobenzene

$$
\begin{aligned}
& \text { Crystal data } \\
& \mathrm{C}_{6} \mathrm{H}_{2} \mathrm{Br}_{3} \mathrm{NO}_{2} \\
& M_{r}=359.82 \\
& \text { Triclinic, } P \overline{1} \\
& a=7.641(5) \AA \\
& b=8.040(5) \AA \\
& c=14.917(6) \AA \\
& \alpha=83.91(3)^{\circ} \\
& \beta=79.86(4)^{\circ} \\
& \gamma=81.49(4)^{\circ} \\
& V=889.2(8) \AA^{3} \\
& Z=4
\end{aligned}
$$

\section{Data collection}

Bruker P4

diffractometer

Radiation source: fine-focus sealed tube

Graphite monochromator

$\omega$ scans

Absorption correction: part of the refinement model $(\Delta F)$

(Walker \& Stuart, 1983)

$T_{\min }=0.0002, T_{\max }=0.001$

6070 measured reflections

\section{Refinement}

Refinement on $F^{2}$

Least-squares matrix: full

$R\left[F^{2}>2 \sigma\left(F^{2}\right)\right]=0.066$

$w R\left(F^{2}\right)=0.196$

$S=1.47$

3141 reflections
$F(000)=664$

$D_{\mathrm{x}}=2.688 \mathrm{Mg} \mathrm{m}^{-3}$

Melting point: $380 \mathrm{~K}$

Mo $K \alpha$ radiation, $\lambda=0.71073 \AA$

Cell parameters from 48 reflections

$\theta=4.8-12.4^{\circ}$

$\mu=13.57 \mathrm{~mm}^{-1}$

$T=298 \mathrm{~K}$

Irregular, colourless

$0.42 \times 0.40 \times 0.30 \mathrm{~mm}$

3141 independent reflections

1503 reflections with $I>2 \sigma(I)$

$R_{\text {int }}=0.120$

$\theta_{\max }=25.1^{\circ}, \theta_{\min }=2.6^{\circ}$

$h=-8 \rightarrow 9$

$k=-9 \rightarrow 9$

$l=0 \rightarrow 17$

3 standard reflections every 97 reflections

intensity decay: $1 \%$

\author{
218 parameters \\ 0 restraints \\ 0 constraints \\ Primary atom site location: structure-invariant \\ direct methods
}


Secondary atom site location: difference Fourier map

Hydrogen site location: inferred from neighbouring sites

$\mathrm{H}$-atom parameters constrained

$w=1 /\left[\sigma^{2}\left(F_{\mathrm{o}}^{2}\right)+(0.050 P)^{2}\right]$

where $P=\left(F_{\mathrm{o}}^{2}+2 F_{\mathrm{c}}^{2}\right) / 3$

$$
\begin{aligned}
& (\Delta / \sigma)_{\max }<0.001 \\
& \Delta \rho_{\max }=0.79 \mathrm{e} \AA^{-3} \\
& \Delta \rho_{\min }=-1.00 \mathrm{e} \AA^{-3} \\
& \text { Extinction correction: SHELXL2014 } \\
& \quad(\text { Sheldrick, } 2015), \\
& \quad \mathrm{Fc}^{*}=\mathrm{kFc}\left[1+0.001 \times \mathrm{xc}^{2} \lambda^{3} / \sin (2 \theta)\right]^{-1 / 4} \\
& \text { Extinction coefficient: } 0.0063(12)
\end{aligned}
$$

\begin{tabular}{|c|c|c|c|c|}
\hline & $x$ & $y$ & $z$ & $U_{\text {iso }} * / U_{\text {eq }}$ \\
\hline $\mathrm{Br} 1$ & $0.1438(2)$ & $0.3194(2)$ & $0.05082(13)$ & $0.0863(6)$ \\
\hline $\mathrm{Br} 2$ & $0.2958(2)$ & $0.0254(2)$ & $0.20089(11)$ & $0.0855(6)$ \\
\hline Br3 & $0.4312(2)$ & $-0.3604(2)$ & $0.13778(10)$ & $0.0791(5)$ \\
\hline $\mathrm{C} 1$ & $0.2177(18)$ & 0.1018 (19) & $0.0215(9)$ & $0.070(4)$ \\
\hline $\mathrm{C} 2$ & $0.2831(17)$ & $-0.0235(17)$ & $0.0860(9)$ & $0.063(3)$ \\
\hline $\mathrm{C} 3$ & $0.3360(14)$ & $-0.1910(16)$ & $0.0555(8)$ & $0.056(3)$ \\
\hline $\mathrm{C} 4$ & $0.3227(18)$ & $-0.2259(19)$ & $-0.0296(9)$ & $0.069(4)$ \\
\hline $\mathrm{H} 4$ & 0.3573 & -0.3355 & -0.0465 & $0.083 *$ \\
\hline $\mathrm{C} 5$ & $0.2615(17)$ & $-0.1072(16)$ & $-0.0895(10)$ & $0.064(3)$ \\
\hline C6 & $0.2067(16)$ & $0.0562(16)$ & $-0.0661(9)$ & $0.059(3)$ \\
\hline H6 & 0.1619 & 0.1373 & -0.1085 & $0.071 *$ \\
\hline N1 & $0.2502(16)$ & $-0.1453(18)$ & $-0.1809(8)$ & $0.075(3)$ \\
\hline $\mathrm{O} 1$ & $0.2967(16)$ & $-0.2925(16)$ & $-0.2012(7)$ & $0.094(3)$ \\
\hline $\mathrm{O} 2$ & $0.1923(18)$ & $-0.0342(15)$ & $-0.2318(8)$ & $0.105(4)$ \\
\hline Br11 & $0.3943(2)$ & $0.2012(2)$ & $0.39885(13)$ & $0.0891(6)$ \\
\hline Br12 & $0.1013(2)$ & $0.1170(2)$ & $0.58586(10)$ & $0.0795(6)$ \\
\hline Br13 & $-0.3231(2)$ & $0.2845(2)$ & $0.59130(11)$ & $0.0865(6)$ \\
\hline C11 & $0.151(2)$ & $0.2912(18)$ & $0.4092(12)$ & $0.077(4)$ \\
\hline $\mathrm{C} 12$ & $0.0303(18)$ & $0.2505(18)$ & $0.4860(10)$ & $0.064(3)$ \\
\hline $\mathrm{C} 13$ & $-0.150(2)$ & $0.323(2)$ & $0.4913(9)$ & $0.073(4)$ \\
\hline $\mathrm{C} 14$ & $-0.2002(19)$ & 0.4188 (19) & $0.4208(9)$ & $0.070(4)$ \\
\hline H14 & -0.3207 & 0.4619 & 0.4232 & $0.084 *$ \\
\hline $\mathrm{C} 15$ & $-0.079(2)$ & $0.4583(18)$ & $0.3427(8)$ & $0.068(4)$ \\
\hline $\mathrm{C} 16$ & 0.0943 (19) & $0.3923(18)$ & $0.3375(9)$ & $0.068(4)$ \\
\hline H16 & 0.1756 & 0.4155 & 0.2850 & $0.082 *$ \\
\hline N11 & $-0.1327(18)$ & $0.5801(16)$ & $0.2644(10)$ & $0.076(3)$ \\
\hline $\mathrm{O} 11$ & $-0.2882(14)$ & $0.6432(13)$ & $0.2757(7)$ & $0.083(3)$ \\
\hline $\mathrm{O} 12$ & $-0.0224(17)$ & $0.5952(18)$ & $0.1956(8)$ & $0.110(4)$ \\
\hline
\end{tabular}

Fractional atomic coordinates and isotropic or equivalent isotropic displacement parameters $\left(\AA^{2}\right)$

Atomic displacement parameters $\left(\AA^{2}\right)$

\begin{tabular}{lllllll}
\hline & $U^{11}$ & $U^{22}$ & $U^{33}$ & $U^{12}$ & $U^{13}$ & $U^{23}$ \\
\hline Br1 & $0.0776(11)$ & $0.0671(10)$ & $0.1094(12)$ & $-0.0047(8)$ & $-0.0022(9)$ & $-0.0140(8)$ \\
Br2 & $0.0876(11)$ & $0.0974(13)$ & $0.0716(9)$ & $-0.0124(9)$ & $-0.0075(8)$ & $-0.0164(8)$ \\
Br3 & $0.0765(10)$ & $0.0789(11)$ & $0.0793(10)$ & $-0.0043(8)$ & $-0.0186(8)$ & $0.0070(8)$ \\
C1 & $0.062(8)$ & $0.078(10)$ & $0.072(9)$ & $-0.013(7)$ & $-0.022(7)$ & $0.001(7)$ \\
C2 & $0.058(8)$ & $0.068(9)$ & $0.062(8)$ & $-0.006(7)$ & $0.004(6)$ & $-0.026(7)$ \\
C3 & $0.034(6)$ & $0.064(8)$ & $0.060(7)$ & $-0.001(6)$ & $0.001(6)$ & $0.012(6)$
\end{tabular}




\begin{tabular}{lllllll} 
C4 & $0.063(8)$ & $0.072(9)$ & $0.071(9)$ & $0.022(7)$ & $-0.018(7)$ & $-0.030(7)$ \\
C5 & $0.056(8)$ & $0.051(8)$ & $0.087(10)$ & $-0.015(6)$ & $-0.021(7)$ & $0.009(7)$ \\
C6 & $0.062(8)$ & $0.052(8)$ & $0.073(8)$ & $-0.019(6)$ & $-0.033(7)$ & $0.003(6)$ \\
N1 & $0.079(8)$ & $0.081(9)$ & $0.080(8)$ & $-0.016(7)$ & $-0.036(7)$ & $-0.026(7)$ \\
O1 & $0.119(9)$ & $0.095(9)$ & $0.071(6)$ & $-0.005(7)$ & $-0.017(6)$ & $-0.032(6)$ \\
O2 & $0.151(11)$ & $0.087(9)$ & $0.089(7)$ & $-0.010(8)$ & $-0.061(8)$ & $0.004(6)$ \\
Br11 & $0.0644(10)$ & $0.0993(13)$ & $0.1026(12)$ & $0.0018(9)$ & $-0.0185(9)$ & $-0.0137(10)$ \\
Br12 & $0.0955(12)$ & $0.0701(10)$ & $0.0769(9)$ & $-0.0090(8)$ & $-0.0294(9)$ & $-0.0017(7)$ \\
Br13 & $0.0785(11)$ & $0.0945(13)$ & $0.0808(10)$ & $-0.0202(9)$ & $0.0012(8)$ & $0.0067(9)$ \\
C11 & $0.084(10)$ & $0.050(8)$ & $0.102(11)$ & $-0.013(7)$ & $-0.021(9)$ & $-0.015(8)$ \\
C12 & $0.060(8)$ & $0.062(8)$ & $0.073(9)$ & $-0.018(7)$ & $-0.012(8)$ & $-0.008(7)$ \\
C13 & $0.081(10)$ & $0.082(10)$ & $0.060(8)$ & $-0.020(8)$ & $-0.024(7)$ & $0.009(7)$ \\
C14 & $0.056(8)$ & $0.080(10)$ & $0.065(8)$ & $-0.010(7)$ & $0.011(7)$ & $-0.007(7)$ \\
C15 & $0.088(10)$ & $0.077(10)$ & $0.040(6)$ & $0.019(8)$ & $-0.034(7)$ & $-0.004(6)$ \\
C16 & $0.070(9)$ & $0.072(9)$ & $0.062(8)$ & $-0.028(8)$ & $0.006(7)$ & $-0.003(7)$ \\
N11 & $0.067(8)$ & $0.065(8)$ & $0.093(10)$ & $-0.005(6)$ & $-0.017(7)$ & $0.011(7)$ \\
O11 & $0.074(7)$ & $0.079(7)$ & $0.099(7)$ & $0.003(6)$ & $-0.032(6)$ & $-0.014(6)$ \\
O12 & $0.097(8)$ & $0.144(12)$ & $0.077(7)$ & $-0.012(8)$ & $-0.011(7)$ & $0.028(7)$ \\
& & & & & & \\
\hline
\end{tabular}

Geometric parameters $\left(\AA,{ }^{\circ}\right)$

\begin{tabular}{llll}
\hline $\mathrm{Br} 1-\mathrm{C} 1$ & $1.831(15)$ & $\mathrm{Br} 11-\mathrm{C} 11$ & $1.877(15)$ \\
$\mathrm{Br} 2-\mathrm{C} 2$ & $1.821(12)$ & $\mathrm{B} 12-\mathrm{C} 12$ & $1.854(14)$ \\
$\mathrm{Br} 3-\mathrm{C} 3$ & $1.886(11)$ & $\mathrm{B} 13-\mathrm{C} 13$ & $1.842(15)$ \\
$\mathrm{C} 1-\mathrm{C} 6$ & $1.415(18)$ & $\mathrm{C} 11-\mathrm{C} 16$ & $1.368(19)$ \\
$\mathrm{C} 1-\mathrm{C} 2$ & $1.416(18)$ & $\mathrm{C} 11-\mathrm{C} 12$ & $1.38(2)$ \\
$\mathrm{C} 2-\mathrm{C} 3$ & $1.445(18)$ & $\mathrm{C} 12-\mathrm{C} 13$ & $1.410(19)$ \\
$\mathrm{C} 3-\mathrm{C} 4$ & $1.353(17)$ & $\mathrm{C} 13-\mathrm{C} 14$ & $1.313(18)$ \\
$\mathrm{C} 4-\mathrm{C} 5$ & $1.328(17)$ & $\mathrm{C} 14-\mathrm{C} 15$ & $1.39(2)$ \\
$\mathrm{C} 4-\mathrm{H} 4$ & 0.9300 & $\mathrm{C} 14-\mathrm{H} 14$ & 0.9300 \\
$\mathrm{C} 5-\mathrm{C} 6$ & $1.381(19)$ & $\mathrm{C} 15-\mathrm{C} 16$ & $1.347(19)$ \\
$\mathrm{C} 5-\mathrm{N} 1$ & $1.448(18)$ & $\mathrm{C} 15-\mathrm{N} 11$ & $1.515(16)$ \\
$\mathrm{C} 6-\mathrm{H} 6$ & 0.9300 & $\mathrm{C} 16-\mathrm{H} 16$ & 0.9300 \\
$\mathrm{~N} 1-\mathrm{O} 2$ & $1.194(15)$ & $\mathrm{N} 11-\mathrm{O} 11$ & $1.211(15)$ \\
$\mathrm{N} 1-\mathrm{O} 1$ & $1.238(16)$ & $\mathrm{N} 11-\mathrm{O} 12$ & $1216(16)$ \\
& & & $118.6(14)$ \\
$\mathrm{C} 6-\mathrm{C} 1-\mathrm{C} 2$ & $119.0(13)$ & $\mathrm{C} 16-\mathrm{C} 11-\mathrm{C} 12$ & $120.9(11)$ \\
$\mathrm{C} 6-\mathrm{C} 1-\mathrm{Br} 1$ & $119.9(9)$ & $\mathrm{C} 16-\mathrm{C} 11-\mathrm{Br} 11$ & $118.8(13)$ \\
$\mathrm{C} 2-\mathrm{C} 1-\mathrm{Br} 1$ & $121.0(10)$ & $\mathrm{C} 12-\mathrm{C} 11-\mathrm{Br} 11$ & $122.1(10)$ \\
$\mathrm{C} 1-\mathrm{C} 2-\mathrm{C} 3$ & $116.2(11)$ & $\mathrm{C} 11-\mathrm{C} 12-\mathrm{C} 13$ & $118.9(10)$ \\
$\mathrm{C} 1-\mathrm{C} 2-\mathrm{Br} 2$ & $121.3(10)$ & $\mathrm{C} 11-\mathrm{C} 12-\mathrm{Br} 12$ & $118.8(14)$ \\
$\mathrm{C} 3-\mathrm{C} 2-\mathrm{Br} 2$ & $122.5(9)$ & $\mathrm{C} 13-\mathrm{C} 12-\mathrm{Br} 12$ & $118.0(11)$ \\
$\mathrm{C} 4-\mathrm{C} 3-\mathrm{C} 2$ & $121.8(11)$ & $\mathrm{C} 14-\mathrm{C} 13-\mathrm{C} 12$ & $123.1(10)$ \\
$\mathrm{C} 4-\mathrm{C} 3-\mathrm{Br} 3$ & $120.6(10)$ & $\mathrm{C} 14-\mathrm{C} 13-\mathrm{Br} 13$ & $122.5(13)$ \\
$\mathrm{C} 2-\mathrm{C} 3-\mathrm{Br} 3$ & $117.6(9)$ & $\mathrm{C} 12-\mathrm{C} 13-\mathrm{B} 13$ & 118.8 \\
$\mathrm{C} 5-\mathrm{C} 4-\mathrm{C} 3$ & $121.5(13)$ & $\mathrm{C} 13-\mathrm{C} 14-\mathrm{C} 15$ & \\
$\mathrm{C} 5-\mathrm{C} 4-\mathrm{H} 4$ & 119.3 & $\mathrm{C} 13-\mathrm{C} 14-\mathrm{H} 14$ & $\mathrm{C} 15-\mathrm{C} 14-\mathrm{H} 14$ \\
$\mathrm{C} 3-\mathrm{C} 4-\mathrm{H} 4$ & 119.3 & & \\
& & &
\end{tabular}




\begin{tabular}{|c|c|c|c|}
\hline $\mathrm{C} 4-\mathrm{C} 5-\mathrm{C} 6$ & $120.7(13)$ & $\mathrm{C} 16-\mathrm{C} 15-\mathrm{C} 14$ & $119.2(11)$ \\
\hline $\mathrm{C} 4-\mathrm{C} 5-\mathrm{N} 1$ & $121.1(13)$ & $\mathrm{C} 16-\mathrm{C} 15-\mathrm{N} 11$ & $117.9(13)$ \\
\hline $\mathrm{C} 6-\mathrm{C} 5-\mathrm{N} 1$ & $118.2(11)$ & $\mathrm{C} 14-\mathrm{C} 15-\mathrm{N} 11$ & $122.8(12)$ \\
\hline $\mathrm{C} 5-\mathrm{C} 6-\mathrm{C} 1$ & $120.8(11)$ & $\mathrm{C} 15-\mathrm{C} 16-\mathrm{C} 11$ & $120.0(14)$ \\
\hline $\mathrm{C} 5-\mathrm{C} 6-\mathrm{H} 6$ & 119.6 & $\mathrm{C} 15-\mathrm{C} 16-\mathrm{H} 16$ & 120.0 \\
\hline $\mathrm{C} 1-\mathrm{C} 6-\mathrm{H} 6$ & 119.6 & $\mathrm{C} 11-\mathrm{C} 16-\mathrm{H} 16$ & 120.0 \\
\hline $\mathrm{O} 2-\mathrm{N} 1-\mathrm{O} 1$ & $123.6(12)$ & $\mathrm{O} 11-\mathrm{N} 11-\mathrm{O} 12$ & $127.0(13)$ \\
\hline $\mathrm{O} 2-\mathrm{N} 1-\mathrm{C} 5$ & $118.3(13)$ & $\mathrm{O} 11-\mathrm{N} 11-\mathrm{C} 15$ & $114.7(13)$ \\
\hline $\mathrm{O} 1-\mathrm{N} 1-\mathrm{C} 5$ & $118.1(12)$ & $\mathrm{O} 12-\mathrm{N} 11-\mathrm{C} 15$ & $118.1(12)$ \\
\hline $\mathrm{C} 6-\mathrm{C} 1-\mathrm{C} 2-\mathrm{C} 3$ & $0.6(19)$ & $\mathrm{C} 16-\mathrm{C} 11-\mathrm{C} 12-\mathrm{C} 13$ & $-4(2)$ \\
\hline $\mathrm{Br} 1-\mathrm{C} 1-\mathrm{C} 2-\mathrm{C} 3$ & $179.5(9)$ & $\mathrm{Br} 11-\mathrm{C} 11-\mathrm{C} 12-\mathrm{C} 13$ & $179.1(11)$ \\
\hline $\mathrm{C} 6-\mathrm{C} 1-\mathrm{C} 2-\mathrm{Br} 2$ & $-178.5(10)$ & $\mathrm{C} 16-\mathrm{C} 11-\mathrm{C} 12-\mathrm{Br} 12$ & $-178.9(11)$ \\
\hline $\mathrm{Br} 1-\mathrm{C} 1-\mathrm{C} 2-\mathrm{Br} 2$ & $0.3(16)$ & $\mathrm{Br} 11-\mathrm{C} 11-\mathrm{C} 12-\mathrm{Br} 12$ & $4.0(17)$ \\
\hline $\mathrm{C} 1-\mathrm{C} 2-\mathrm{C} 3-\mathrm{C} 4$ & $-0.3(19)$ & $\mathrm{C} 11-\mathrm{C} 12-\mathrm{C} 13-\mathrm{C} 14$ & $4(2)$ \\
\hline $\mathrm{Br} 2-\mathrm{C} 2-\mathrm{C} 3-\mathrm{C} 4$ & $178.8(11)$ & $\mathrm{Br} 12-\mathrm{C} 12-\mathrm{C} 13-\mathrm{C} 14$ & $179.4(12)$ \\
\hline $\mathrm{C} 1-\mathrm{C} 2-\mathrm{C} 3-\mathrm{Br} 3$ & $178.0(9)$ & $\mathrm{C} 11-\mathrm{C} 12-\mathrm{C} 13-\mathrm{Br} 13$ & $-178.0(11)$ \\
\hline $\mathrm{Br} 2-\mathrm{C} 2-\mathrm{C} 3-\mathrm{Br} 3$ & $-2.8(14)$ & $\mathrm{Br} 12-\mathrm{C} 12-\mathrm{C} 13-\mathrm{Br} 13$ & $-2.7(18)$ \\
\hline $\mathrm{C} 2-\mathrm{C} 3-\mathrm{C} 4-\mathrm{C} 5$ & $1(2)$ & $\mathrm{C} 12-\mathrm{C} 13-\mathrm{C} 14-\mathrm{C} 15$ & $-3(2)$ \\
\hline $\mathrm{Br} 3-\mathrm{C} 3-\mathrm{C} 4-\mathrm{C} 5$ & $-177.8(11)$ & $\mathrm{Br} 13-\mathrm{C} 13-\mathrm{C} 14-\mathrm{C} 15$ & $178.7(12)$ \\
\hline $\mathrm{C} 3-\mathrm{C} 4-\mathrm{C} 5-\mathrm{C} 6$ & $-1(2)$ & $\mathrm{C} 13-\mathrm{C} 14-\mathrm{C} 15-\mathrm{C} 16$ & $2(2)$ \\
\hline $\mathrm{C} 3-\mathrm{C} 4-\mathrm{C} 5-\mathrm{N} 1$ & $179.1(13)$ & $\mathrm{C} 13-\mathrm{C} 14-\mathrm{C} 15-\mathrm{N} 11$ & $-174.8(15)$ \\
\hline $\mathrm{C} 4-\mathrm{C} 5-\mathrm{C} 6-\mathrm{C} 1$ & $1(2)$ & $\mathrm{C} 14-\mathrm{C} 15-\mathrm{C} 16-\mathrm{C} 11$ & $-2(2)$ \\
\hline $\mathrm{N} 1-\mathrm{C} 5-\mathrm{C} 6-\mathrm{C} 1$ & $-178.8(12)$ & $\mathrm{N} 11-\mathrm{C} 15-\mathrm{C} 16-\mathrm{C} 11$ & $175.4(13)$ \\
\hline $\mathrm{C} 2-\mathrm{C} 1-\mathrm{C} 6-\mathrm{C} 5$ & $-1(2)$ & $\mathrm{C} 12-\mathrm{C} 11-\mathrm{C} 16-\mathrm{C} 15$ & $3(2)$ \\
\hline $\mathrm{Br} 1-\mathrm{C} 1-\mathrm{C} 6-\mathrm{C} 5$ & $-180.0(10)$ & $\mathrm{Br} 11-\mathrm{C} 11-\mathrm{C} 16-\mathrm{C} 15$ & $179.8(11)$ \\
\hline $\mathrm{C} 4-\mathrm{C} 5-\mathrm{N} 1-\mathrm{O} 2$ & $179.2(14)$ & $\mathrm{C} 16-\mathrm{C} 15-\mathrm{N} 11-\mathrm{O} 11$ & $-175.5(13)$ \\
\hline $\mathrm{C} 6-\mathrm{C} 5-\mathrm{N} 1-\mathrm{O} 2$ & $-1(2)$ & $\mathrm{C} 14-\mathrm{C} 15-\mathrm{N} 11-\mathrm{O} 11$ & $1(2)$ \\
\hline $\mathrm{C} 4-\mathrm{C} 5-\mathrm{N} 1-\mathrm{O} 1$ & $1(2)$ & $\mathrm{C} 16-\mathrm{C} 15-\mathrm{N} 11-\mathrm{O} 12$ & $10(2)$ \\
\hline $\mathrm{C} 6-\mathrm{C} 5-\mathrm{N} 1-\mathrm{O} 1$ & $-178.8(13)$ & $\mathrm{C} 14-\mathrm{C} 15-\mathrm{N} 11-\mathrm{O} 12$ & $-173.5(15)$ \\
\hline
\end{tabular}

(II) 1,3-Dibromo-2-iodo-5-nitrobenzene

Crystal data

$\mathrm{C}_{6} \mathrm{H}_{2} \mathrm{Br}_{2} \mathrm{INO}_{2}$

$M_{r}=406.81$

Monoclinic, $P 2_{1} / c$

$a=13.548(3) \AA$

$b=20.037(3) \AA$

$c=9.123(2) \AA$

$\beta=130.37(2)^{\circ}$

$V=1886.8(8) \AA^{3}$

$Z=8$

$F(000)=1472$

\section{Data collection}

Bruker P4

diffractometer

Radiation source: fine-focus sealed tube

Graphite monochromator
$D_{\mathrm{x}}=2.864 \mathrm{Mg} \mathrm{m}^{-3}$

Melting point: $415 \mathrm{~K}$

Mo $K \alpha$ radiation, $\lambda=0.71073 \AA$

Cell parameters from 43 reflections

$\theta=5.7-12.5^{\circ}$

$\mu=11.82 \mathrm{~mm}^{-1}$

$T=298 \mathrm{~K}$

Prism, brown

$0.50 \times 0.22 \times 0.12 \mathrm{~mm}$

$2 \theta / \omega$ scans

Absorption correction: $\psi$ scan

(XSCANS; Bruker, 1997)

$T_{\min }=0.429, T_{\max }=0.988$ 
5716 measured reflections

5407 independent reflections

1968 reflections with $I>2 \sigma(I)$

$R_{\text {int }}=0.058$

$\theta_{\text {max }}=30.0^{\circ}, \theta_{\text {min }}=2.2^{\circ}$

\section{Refinement}

Refinement on $F^{2}$

Least-squares matrix: full

$R\left[F^{2}>2 \sigma\left(F^{2}\right)\right]=0.061$

$w R\left(F^{2}\right)=0.153$

$S=0.95$

5407 reflections

218 parameters

0 restraints

0 constraints

Primary atom site location: structure-invariant direct methods

Secondary atom site location: difference Fourier map $h=-14 \rightarrow 19$

$k=0 \rightarrow 28$

$l=-12 \rightarrow 0$

3 standard reflections every 97 reflections

intensity decay: $1 \%$

Hydrogen site location: inferred from neighbouring sites

$\mathrm{H}$-atom parameters constrained

$w=1 /\left[\sigma^{2}\left(F_{\mathrm{o}}^{2}\right)+(0.053 P)^{2}\right]$

where $P=\left(F_{\mathrm{o}}^{2}+2 F_{\mathrm{c}}^{2}\right) / 3$

$(\Delta / \sigma)_{\max }<0.001$

$\Delta \rho_{\max }=0.84$ e $\AA^{-3}$

$\Delta \rho_{\min }=-0.84$ e $\AA^{-3}$

Extinction correction: SHELXL2014 (Sheldrick, 2015), $\mathrm{Fc}^{*}=\mathrm{kFc}\left[1+0.001 \mathrm{xFc}^{2} \lambda^{3} / \sin (2 \theta)\right]^{-1 / 4}$

Extinction coefficient: 0.00093 (11)

Fractional atomic coordinates and isotropic or equivalent isotropic displacement parameters $\left(\AA^{2}\right)$

\begin{tabular}{|c|c|c|c|c|}
\hline & $x$ & $y$ & $z$ & $U_{\text {iso }} * / U_{\text {eq }}$ \\
\hline $\mathrm{Br} 1$ & $0.30362(13)$ & $0.43988(8)$ & $0.2401(2)$ & $0.0615(4)$ \\
\hline $\mathrm{I} 2$ & $0.54556(10)$ & $0.36925(4)$ & $0.26460(13)$ & $0.0583(3)$ \\
\hline $\mathrm{Br} 3$ & $0.73278(12)$ & $0.49074(7)$ & $0.26614(18)$ & $0.0561(4)$ \\
\hline $\mathrm{C} 1$ & $0.4175(11)$ & $0.4967(7)$ & $0.2453(16)$ & $0.035(3)$ \\
\hline $\mathrm{C} 2$ & $0.5191(11)$ & $0.4723(6)$ & $0.2535(13)$ & $0.033(3)$ \\
\hline $\mathrm{C} 3$ & $0.5960(11)$ & $0.5182(6)$ & $0.2557(17)$ & $0.038(3)$ \\
\hline $\mathrm{C} 4$ & $0.5754(12)$ & $0.5862(6)$ & $0.2478(17)$ & $0.040(3)$ \\
\hline $\mathrm{H} 4 \mathrm{~A}$ & 0.6264 & 0.6171 & 0.2474 & $0.048 *$ \\
\hline C5 & $0.4763(13)$ & $0.6050(7)$ & $0.2405(15)$ & $0.046(4)$ \\
\hline C6 & $0.3965(12)$ & $0.5642(7)$ & $0.2397(18)$ & $0.048(4)$ \\
\hline H6A & 0.3307 & 0.5809 & 0.2355 & $0.057 *$ \\
\hline N1 & $0.4555(13)$ & $0.6807(6)$ & $0.2380(17)$ & $0.065(3)$ \\
\hline $\mathrm{O} 1$ & $0.5145(11)$ & $0.7161(5)$ & $0.2088(17)$ & $0.085(4)$ \\
\hline $\mathrm{O} 2$ & $0.3795(15)$ & $0.6989(5)$ & $0.2557(18)$ & $0.105(4)$ \\
\hline Br11 & $-0.18918(13)$ & $0.30721(8)$ & $0.2488(2)$ & $0.0620(4)$ \\
\hline $\mathrm{I} 12$ & $0.04866(10)$ & $0.37922(4)$ & $0.26639(13)$ & $0.0593(3)$ \\
\hline Br13 & $0.24427(12)$ & $0.25893(8)$ & $0.2825(2)$ & 0.0588 \\
\hline C11 & $-0.0736(11)$ & $0.2509(7)$ & $0.2562(17)$ & $0.039(3)$ \\
\hline $\mathrm{C} 12$ & $0.0237(12)$ & $0.2769(7)$ & $0.2619(14)$ & $0.035(3)$ \\
\hline $\mathrm{C} 13$ & $0.1046(11)$ & $0.2312(6)$ & $0.2672(18)$ & $0.039(3)$ \\
\hline $\mathrm{C} 14$ & $0.0869(13)$ & $0.1639(6)$ & $0.2665(18)$ & $0.045(4)$ \\
\hline $\mathrm{H} 14 \mathrm{~A}$ & 0.1429 & 0.1344 & 0.2736 & $0.054 *$ \\
\hline C15 & $-0.0137(13)$ & $0.1391(8)$ & $0.2553(16)$ & $0.045(4)$ \\
\hline C16 & $-0.0922(13)$ & $0.1829(6)$ & $0.2507(18)$ & $0.043(4)$ \\
\hline H16A & -0.1596 & 0.1668 & 0.2437 & $0.052 *$ \\
\hline N11 & $-0.0302(15)$ & $0.0681(6)$ & $0.2483(17)$ & $0.066(4)$ \\
\hline O11 & $0.0350(12)$ & $0.0323(6)$ & $0.2418(18)$ & $0.099(4)$ \\
\hline
\end{tabular}


Atomic displacement parameters $\left(\AA^{2}\right)$

\begin{tabular}{lllllll}
\hline & $U^{11}$ & $U^{22}$ & $U^{33}$ & $U^{12}$ & $U^{13}$ & $U^{23}$ \\
\hline $\mathrm{Br} 1$ & $0.0558(8)$ & $0.0680(9)$ & $0.0684(9)$ & $-0.0167(7)$ & $0.0437(7)$ & $-0.0035(7)$ \\
$\mathrm{I} 2$ & $0.0737(6)$ & $0.0268(4)$ & $0.0723(7)$ & $0.0048(5)$ & $0.0464(5)$ & $0.0017(4)$ \\
$\mathrm{Br} 3$ & $0.0484(7)$ & $0.0636(9)$ & $0.0654(9)$ & $0.0047(7)$ & $0.0409(7)$ & $0.0002(7)$ \\
$\mathrm{C} 1$ & $0.041(7)$ & $0.035(7)$ & $0.033(6)$ & $-0.012(6)$ & $0.027(5)$ & $-0.007(5)$ \\
$\mathrm{C} 2$ & $0.043(7)$ & $0.017(6)$ & $0.035(8)$ & $0.002(5)$ & $0.024(6)$ & $0.000(4)$ \\
$\mathrm{C} 3$ & $0.042(6)$ & $0.033(7)$ & $0.041(7)$ & $0.003(5)$ & $0.028(6)$ & $-0.004(5)$ \\
$\mathrm{C} 4$ & $0.037(7)$ & $0.036(8)$ & $0.048(8)$ & $-0.005(6)$ & $0.027(6)$ & $-0.003(6)$ \\
$\mathrm{C} 5$ & $0.046(7)$ & $0.023(7)$ & $0.040(8)$ & $0.005(6)$ & $0.015(6)$ & $-0.002(5)$ \\
$\mathrm{C} 6$ & $0.036(7)$ & $0.064(10)$ & $0.045(8)$ & $-0.003(7)$ & $0.027(6)$ & $-0.016(7)$ \\
$\mathrm{N} 1$ & $0.066(8)$ & $0.031(7)$ & $0.075(9)$ & $0.010(7)$ & $0.036(7)$ & $-0.001(6)$ \\
O1 & $0.091(8)$ & $0.030(6)$ & $0.108(9)$ & $-0.003(6)$ & $0.053(7)$ & $0.003(6)$ \\
O2 & $0.163(12)$ & $0.050(7)$ & $0.137(11)$ & $0.050(8)$ & $0.112(10)$ & $0.016(6)$ \\
$\mathrm{Br} 11$ & $0.0522(8)$ & $0.0681(9)$ & $0.0685(9)$ & $0.0108(7)$ & $0.0404(7)$ & $-0.0033(7)$ \\
$\mathrm{I} 12$ & $0.0755(6)$ & $0.0283(4)$ & $0.0735(7)$ & $-0.0069(5)$ & $0.0479(5)$ & $-0.0038(4)$ \\
$\mathrm{Br} 13$ & $0.0488(7)$ & $0.0631(9)$ & $0.0703(9)$ & $-0.0088(7)$ & $0.0412(7)$ & $-0.0014(7)$ \\
$\mathrm{C} 11$ & $0.043(7)$ & $0.035(7)$ & $0.041(7)$ & $-0.002(6)$ & $0.028(6)$ & $0.002(6)$ \\
$\mathrm{C} 12$ & $0.041(7)$ & $0.033(8)$ & $0.039(8)$ & $0.007(6)$ & $0.030(6)$ & $0.004(4)$ \\
$\mathrm{C} 13$ & $0.033(6)$ & $0.037(8)$ & $0.042(7)$ & $-0.005(5)$ & $0.022(6)$ & $-0.005(6)$ \\
$\mathrm{C} 14$ & $0.051(8)$ & $0.029(7)$ & $0.044(8)$ & $0.008(6)$ & $0.025(6)$ & $0.000(6)$ \\
$\mathrm{C} 15$ & $0.063(9)$ & $0.028(7)$ & $0.056(9)$ & $-0.015(6)$ & $0.045(8)$ & $-0.006(5)$ \\
$\mathrm{C} 16$ & $0.046(7)$ & $0.031(7)$ & $0.042(7)$ & $-0.021(6)$ & $0.024(6)$ & $-0.015(5)$ \\
N11 & $0.096(10)$ & $0.034(7)$ & $0.073(9)$ & $-0.018(7)$ & $0.057(8)$ & $-0.006(6)$ \\
O11 & $0.101(9)$ & $0.029(6)$ & $0.147(11)$ & $-0.010(6)$ & $0.072(8)$ & $-0.005(7)$ \\
O12 & $0.144(11)$ & $0.061(7)$ & $0.130(10)$ & $-0.042(8)$ & $0.106(9)$ & $-0.012(6)$ \\
& & & & & & \\
\hline & & & & & &
\end{tabular}

Geometric parameters $\left(\AA,{ }^{\circ}\right)$

\begin{tabular}{llll}
\hline $\mathrm{Br} 1-\mathrm{C} 1$ & $1.894(12)$ & $\mathrm{Br} 11-\mathrm{C} 11$ & $1.895(14)$ \\
$\mathrm{I}-\mathrm{C} 2$ & $2.088(12)$ & $\mathrm{I} 12-\mathrm{C} 12$ & $2.074(14)$ \\
$\mathrm{Br} 3-\mathrm{C} 3$ & $1.875(13)$ & $\mathrm{Br} 13-\mathrm{C} 13$ & $1.888(13)$ \\
$\mathrm{C} 1-\mathrm{C} 6$ & $1.375(18)$ & $\mathrm{C} 11-\mathrm{C} 12$ & $1.387(17)$ \\
$\mathrm{C} 1-\mathrm{C} 2$ & $1.416(17)$ & $\mathrm{C} 11-\mathrm{C} 16$ & $1.381(18)$ \\
$\mathrm{C} 2-\mathrm{C} 3$ & $1.380(17)$ & $\mathrm{C} 12-\mathrm{C} 13$ & $1.405(17)$ \\
$\mathrm{C} 3-\mathrm{C} 4$ & $1.384(16)$ & $\mathrm{C} 13-\mathrm{C} 14$ & $1.370(16)$ \\
$\mathrm{C} 4-\mathrm{C} 5$ & $1.353(18)$ & $\mathrm{C} 14-\mathrm{C} 15$ & $1.390(19)$ \\
$\mathrm{C} 4-\mathrm{H} 4 \mathrm{~A}$ & 0.9300 & $\mathrm{C} 14-\mathrm{H} 14 \mathrm{~A}$ & 0.9300 \\
$\mathrm{C} 5-\mathrm{C} 6$ & $1.35(2)$ & $\mathrm{C} 15-\mathrm{C} 16$ & $1.359(19)$ \\
$\mathrm{C} 5-\mathrm{N} 1$ & $1.539(18)$ & $\mathrm{C} 15-\mathrm{N} 11$ & $1.435(19)$ \\
$\mathrm{C} 6-\mathrm{H} 6 \mathrm{~A}$ & 0.9300 & $\mathrm{C} 16-\mathrm{H} 16 \mathrm{~A}$ & 0.9300 \\
$\mathrm{~N} 1-\mathrm{O} 2$ & $1.199(17)$ & $\mathrm{N} 11-\mathrm{O} 11$ & $1.168(19)$ \\
$\mathrm{N} 1-\mathrm{O} 1$ & $1.221(19)$ & $\mathrm{N} 11-\mathrm{O} 12$ & $1.226(18)$ \\
$\mathrm{C} 6-\mathrm{C} 1-\mathrm{C} 2$ & & & $121.2(14)$
\end{tabular}




\begin{tabular}{|c|c|c|c|}
\hline $\mathrm{C} 6-\mathrm{C} 1-\mathrm{Br} 1$ & $116.4(11)$ & $\mathrm{C} 12-\mathrm{C} 11-\mathrm{Br} 11$ & $121.4(11)$ \\
\hline $\mathrm{C} 2-\mathrm{C} 1-\mathrm{Br} 1$ & $122.8(10)$ & $\mathrm{C} 16-\mathrm{C} 11-\mathrm{Br} 11$ & $117.3(11)$ \\
\hline $\mathrm{C} 3-\mathrm{C} 2-\mathrm{C} 1$ & $118.1(12)$ & $\mathrm{C} 11-\mathrm{C} 12-\mathrm{C} 13$ & $117.3(13)$ \\
\hline $\mathrm{C} 3-\mathrm{C} 2-\mathrm{I} 2$ & $123.6(10)$ & $\mathrm{C} 11-\mathrm{C} 12-\mathrm{I} 12$ & $120.7(11)$ \\
\hline $\mathrm{C} 1-\mathrm{C} 2-\mathrm{I} 2$ & $118.4(10)$ & $\mathrm{C} 13-\mathrm{C} 12-\mathrm{I} 12$ & $122.0(10)$ \\
\hline $\mathrm{C} 2-\mathrm{C} 3-\mathrm{C} 4$ & $122.0(13)$ & $\mathrm{C} 14-\mathrm{C} 13-\mathrm{C} 12$ & $120.8(13)$ \\
\hline $\mathrm{C} 2-\mathrm{C} 3-\mathrm{Br} 3$ & $121.2(10)$ & $\mathrm{C} 14-\mathrm{C} 13-\mathrm{Br} 13$ & $117.0(11)$ \\
\hline $\mathrm{C} 4-\mathrm{C} 3-\mathrm{Br} 3$ & $116.8(11)$ & $\mathrm{C} 12-\mathrm{C} 13-\mathrm{Br} 13$ & $122.2(10)$ \\
\hline $\mathrm{C} 5-\mathrm{C} 4-\mathrm{C} 3$ & $115.9(13)$ & $\mathrm{C} 13-\mathrm{C} 14-\mathrm{C} 15$ & $120.8(14)$ \\
\hline $\mathrm{C} 5-\mathrm{C} 4-\mathrm{H} 4 \mathrm{~A}$ & 122.0 & $\mathrm{C} 13-\mathrm{C} 14-\mathrm{H} 14 \mathrm{~A}$ & 119.6 \\
\hline $\mathrm{C} 3-\mathrm{C} 4-\mathrm{H} 4 \mathrm{~A}$ & 122.0 & $\mathrm{C} 15-\mathrm{C} 14-\mathrm{H} 14 \mathrm{~A}$ & 119.6 \\
\hline $\mathrm{C} 4-\mathrm{C} 5-\mathrm{C} 6$ & $126.6(14)$ & $\mathrm{C} 16-\mathrm{C} 15-\mathrm{C} 14$ & $118.8(14)$ \\
\hline $\mathrm{C} 4-\mathrm{C} 5-\mathrm{N} 1$ & $116.2(15)$ & $\mathrm{C} 16-\mathrm{C} 15-\mathrm{N} 11$ & $122.8(14)$ \\
\hline $\mathrm{C} 6-\mathrm{C} 5-\mathrm{N} 1$ & $117.2(15)$ & $\mathrm{C} 14-\mathrm{C} 15-\mathrm{N} 11$ & $118.3(15)$ \\
\hline $\mathrm{C} 5-\mathrm{C} 6-\mathrm{C} 1$ & $116.7(13)$ & $\mathrm{C} 15-\mathrm{C} 16-\mathrm{C} 11$ & $121.0(14)$ \\
\hline $\mathrm{C} 5-\mathrm{C} 6-\mathrm{H} 6 \mathrm{~A}$ & 121.7 & $\mathrm{C} 15-\mathrm{C} 16-\mathrm{H} 16 \mathrm{~A}$ & 119.5 \\
\hline $\mathrm{C} 1-\mathrm{C} 6-\mathrm{H} 6 \mathrm{~A}$ & 121.7 & $\mathrm{C} 11-\mathrm{C} 16-\mathrm{H} 16 \mathrm{~A}$ & 119.5 \\
\hline $\mathrm{O} 2-\mathrm{N} 1-\mathrm{O} 1$ & $126.4(14)$ & $\mathrm{O} 11-\mathrm{N} 11-\mathrm{O} 12$ & $124.2(16)$ \\
\hline $\mathrm{O} 2-\mathrm{N} 1-\mathrm{C} 5$ & $117.6(14)$ & $\mathrm{O} 11-\mathrm{N} 11-\mathrm{C} 15$ & $120.6(18)$ \\
\hline $\mathrm{O} 1-\mathrm{N} 1-\mathrm{C} 5$ & $115.9(17)$ & $\mathrm{O} 12-\mathrm{N} 11-\mathrm{C} 15$ & $115.0(15)$ \\
\hline $\mathrm{C} 6-\mathrm{C} 1-\mathrm{C} 2-\mathrm{C} 3$ & $0.0(16)$ & $\mathrm{C} 16-\mathrm{C} 11-\mathrm{C} 12-\mathrm{C} 13$ & $-1.7(16)$ \\
\hline $\mathrm{Br} 1-\mathrm{C} 1-\mathrm{C} 2-\mathrm{C} 3$ & $179.7(9)$ & $\mathrm{Br} 11-\mathrm{C} 11-\mathrm{C} 12-\mathrm{C} 13$ & $180.0(9)$ \\
\hline $\mathrm{C} 6-\mathrm{C} 1-\mathrm{C} 2-\mathrm{I} 2$ & $179.4(9)$ & $\mathrm{C} 16-\mathrm{C} 11-\mathrm{C} 12-\mathrm{I} 12$ & $179.2(9)$ \\
\hline $\mathrm{Br} 1-\mathrm{C} 1-\mathrm{C} 2-\mathrm{I} 2$ & $-1.0(12)$ & $\mathrm{Br} 11-\mathrm{C} 11-\mathrm{C} 12-\mathrm{I} 12$ & $0.8(13)$ \\
\hline $\mathrm{C} 1-\mathrm{C} 2-\mathrm{C} 3-\mathrm{C} 4$ & $-0.8(17)$ & $\mathrm{C} 11-\mathrm{C} 12-\mathrm{C} 13-\mathrm{C} 14$ & $0.1(17)$ \\
\hline $\mathrm{I} 2-\mathrm{C} 2-\mathrm{C} 3-\mathrm{C} 4$ & $179.9(9)$ & $\mathrm{I} 12-\mathrm{C} 12-\mathrm{C} 13-\mathrm{C} 14$ & $179.2(9)$ \\
\hline $\mathrm{C} 1-\mathrm{C} 2-\mathrm{C} 3-\mathrm{Br} 3$ & $-179.9(9)$ & $\mathrm{C} 11-\mathrm{C} 12-\mathrm{C} 13-\mathrm{Br} 13$ & $-178.2(9)$ \\
\hline $\mathrm{I} 2-\mathrm{C} 2-\mathrm{C} 3-\mathrm{Br} 3$ & $0.7(14)$ & $\mathrm{I} 12-\mathrm{C} 12-\mathrm{C} 13-\mathrm{Br} 13$ & $0.9(14)$ \\
\hline $\mathrm{C} 2-\mathrm{C} 3-\mathrm{C} 4-\mathrm{C} 5$ & $0.9(18)$ & $\mathrm{C} 12-\mathrm{C} 13-\mathrm{C} 14-\mathrm{C} 15$ & $1.7(19)$ \\
\hline $\mathrm{Br} 3-\mathrm{C} 3-\mathrm{C} 4-\mathrm{C} 5$ & $-179.9(9)$ & $\mathrm{Br} 13-\mathrm{C} 13-\mathrm{C} 14-\mathrm{C} 15$ & $-179.9(10)$ \\
\hline $\mathrm{C} 3-\mathrm{C} 4-\mathrm{C} 5-\mathrm{C} 6$ & $-0.2(18)$ & $\mathrm{C} 13-\mathrm{C} 14-\mathrm{C} 15-\mathrm{C} 16$ & $-1.9(18)$ \\
\hline $\mathrm{C} 3-\mathrm{C} 4-\mathrm{C} 5-\mathrm{N} 1$ & $178.2(11)$ & $\mathrm{C} 13-\mathrm{C} 14-\mathrm{C} 15-\mathrm{N} 11$ & $177.8(12)$ \\
\hline $\mathrm{C} 4-\mathrm{C} 5-\mathrm{C} 6-\mathrm{C} 1$ & $-0.5(19)$ & $\mathrm{C} 14-\mathrm{C} 15-\mathrm{C} 16-\mathrm{C} 11$ & $0.3(18)$ \\
\hline $\mathrm{N} 1-\mathrm{C} 5-\mathrm{C} 6-\mathrm{C} 1$ & $-178.9(10)$ & $\mathrm{N} 11-\mathrm{C} 15-\mathrm{C} 16-\mathrm{C} 11$ & $-179.5(12)$ \\
\hline $\mathrm{C} 2-\mathrm{C} 1-\mathrm{C} 6-\mathrm{C} 5$ & $0.6(17)$ & $\mathrm{C} 12-\mathrm{C} 11-\mathrm{C} 16-\mathrm{C} 15$ & $1.6(19)$ \\
\hline $\mathrm{Br} 1-\mathrm{C} 1-\mathrm{C} 6-\mathrm{C} 5$ & $-179.1(9)$ & $\mathrm{Br} 11-\mathrm{C} 11-\mathrm{C} 16-\mathrm{C} 15$ & $180.0(10)$ \\
\hline $\mathrm{C} 4-\mathrm{C} 5-\mathrm{N} 1-\mathrm{O} 2$ & $-171.0(13)$ & $\mathrm{C} 16-\mathrm{C} 15-\mathrm{N} 11-\mathrm{O} 11$ & $175.7(14)$ \\
\hline $\mathrm{C} 6-\mathrm{C} 5-\mathrm{N} 1-\mathrm{O} 2$ & $7.6(18)$ & $\mathrm{C} 14-\mathrm{C} 15-\mathrm{N} 11-\mathrm{O} 11$ & $-4(2)$ \\
\hline $\mathrm{C} 4-\mathrm{C} 5-\mathrm{N} 1-\mathrm{O} 1$ & $12.4(17)$ & $\mathrm{C} 16-\mathrm{C} 15-\mathrm{N} 11-\mathrm{O} 12$ & $-8.3(19)$ \\
\hline $\mathrm{C} 6-\mathrm{C} 5-\mathrm{N} 1-\mathrm{O} 1$ & $-169.1(12)$ & $\mathrm{C} 14-\mathrm{C} 15-\mathrm{N} 11-\mathrm{O} 12$ & $171.9(13)$ \\
\hline
\end{tabular}

